\title{
O uso da acupuntura para dor em pacientes pediátricos oncológicos: revisão integrativa
}

\author{
Use of acupuncture to relieve pain in pediatric cancer patients: integrative review \\ Uso de la acupuntura para alivio del dolor en pacientes pediátricos con cáncer: revisión
}

integradora

Recebido: 14/11/2021 | Revisado: 24/11/2021 | Aceito: 04/01/2022 | Publicado: 05/01/2022

\author{
Cristiane da Silva Varejão \\ ORCID: https://orcid.org/0000-0003-3866-4737 \\ Instituto Nacional do Câncer, Brasil \\ E-mail: cristianevarejao@gmail.com \\ Fátima Helena do Espírito Santo \\ ORCID: https://orcid.org/0000-0003-3866-4737 \\ Universidade Federal Fluminense, Brasil \\ E-mail: fatahelens@gmail.com \\ Maria de Nazaré de Souza Ribeiro \\ ORCID: https://orcid.org/0000-0002-7641-1004 \\ Universidade do Estado do Amazonas, Brasil \\ E-mail: mnribeiro2@gmail.com \\ Marília Fornaciari Grabois \\ ORCID: https://orcid.org/0000-0002-9368-1030 \\ Instituto Nacional do Câncer, Brasil \\ E-mail: mariliagrabois@gmail.com \\ Liliane Faria \\ ORCID: https://orcid.org/0000-0002-9125-1053 \\ Universidade Federal Fluminense, Brasil \\ E-mail: lili.05@hotmail.com \\ Cleisiane Xavier \\ ORCID: https://orcid.org/0000-0003-4689-6204 \\ Universidade do Estado do Amazonas, Brasil \\ E-mail: cxdiniz@gmail.com
}

\begin{abstract}
Resumo
Objetivo: Identificar e analisar as evidências científicas do uso da acupuntura para alívio da dor em pacientes pediátricos oncológicos. Método: Trata-se de uma Revisão Integrativa da literatura. A busca pelos estudos foi realizada em 4 bases de dados relevantes na área da saúde: Medline, Embase, Lilacs e Cinahl. Resultados: Foram identificadas 82 publicações resultantes da busca. Dez foram pré-selecionadas e cinco incluídas na revisão. Evidenciaram-se resultados favoráveis, os quais demonstraram o alívio da dor em crianças e adolescentes com câncer. Considerações finais: Apesar do número pequeno de artigos encontrados, todos apontaram o benefício da acupuntura para alívio da dor. É importante que acupunturistas voltem-se para pesquisas com melhores delineamentos, para que essa terapia complementar possa, através da prática baseada em evidências, ser utilizada como mais uma forma de cuidado, contribuindo dessa forma para uma melhor qualidade de vida dessa clientela.
\end{abstract}

Palavras-chave: Dor; Neoplasias; Criança; Acupuntura.

\begin{abstract}
Objective: Identify and evaluate the scientific evidence for the use of acupuncture for pain relief in pediatric cancer patients. Method: This is an Integrative Review of the literature. This study's search was performed in four relevant databases in health studies field: Medline, Embase, Lilacs and Cinahl. Results: Eighty-two publications were identified in the search. Ten were pre-selected and five were included in the revision. Favorable results showed pain relief in children and teenagers pediatric cancer patients outstand. Final considerations: Despite the small number of articles found, all of them showed the benefits of acupuncture for pain relief. It is important that acupuncturists turn themselves to research with better designs, so this complementary therapy, through evidence-based practices, can be used as another way of care and contribute to a better quality of life of these clients.
\end{abstract}

Keywords: Pain; Neoplasms; Child; Acupuncture.

\section{Resumen}

Objetivo: Identificar y analizar las evidencias científicas del uso de la acupuntura para alivio del dolor en pacientes pediátricos con cáncer. Método: Esta es una revisión integradora de la literatura. La busca por los estudios fue 
realizada en cuatro -bases de datos relevantes en al área de la salud: Medline, Embase, Lilacs y Cinahl. Resultados: Fueran identificadas ochenta y dos publicaciones resultantes de la búsqueda. Diez fueran pre-seleccionadas y cinco incluidas en la revisión. Se evidenciarán resultados favorables a los cuales se han demostrado el alivio en el dolor en niños y adolescentes con cáncer. Consideraciones finales: A pesar de la pequeña cantidad de artículos encontrados, todos han demostrado el beneficio de la acupuntura para el alivio del dolor. Es importante que los acupunturistas se vuelven a la investigación con mejores diseños para que esta terapia complementaria pueda, a través de la práctica basada en la evidencia, ser utilizada como una forma más de atención, contribuyendo así a una mejor calidad de vida para esta clientela.

Palabras clave: Dolor; Neoplasias; Niño; Acupuntura.

\section{Introdução}

O câncer infantil é considerado raro quando comparado com os tumores do adulto, correspondendo entre 1 e $4 \%$ de todos os tumores malignos. Tende a apresentar menores períodos de latência, costuma crescer rapidamente e torna-se bastante invasivo, porém responde melhor à quimioterapia (Ministério da Saúde, 2017). Durante o curso do tratamento o manejo de sintomas decorrentes da doença e da própria terapêutica tem um papel crucial para garantir a qualidade de vida dos pacientes. Crianças com câncer podem ter queixas álgicas durante o diagnóstico, tratamento, progressão de doença e no fim de vida (Tutelman et al 2018).

A dor oncológica pode ser causada por invasão aguda de tecido, inflamação, dor óssea e -neuropática ou uma combinação dessas causas (Gottschling et al., 2014) e seu manejo constitui um desafio complexo para a equipe de saúde que acompanha uma criança ou adolescente com câncer. Crianças com câncer avançado, sem possibilidades de cura, apresentam variados sintomas e sinais decorrentes da progressão da doença. Sintomas físicos debilitantes como dor, anorexia, náuseas, vômitos, fadiga e dispneia são muito comuns, sobretudo na fase de progressão da enfermidade (Pontes \& Kurashima, 2009).

Segundo a última definição da International Association for the Study of Pain (Raja et al., 2020, p.2), a dor é “uma experiência sensitiva e emocional desagradável associada a uma lesão tecidual real ou potencial, ou descrita nos termos de tal lesão". Apresenta como observação (em uma de suas notas) que a dor é sempre uma experiência subjetiva, que é influenciada, em graus variáveis, por fatores biológicos, psicológicos e sociais (Raja et al., 2020).

Os aspectos psicológicos envolvidos na dor também têm papel de destaque na configuração geral do fenômeno. A característica multidimensional da dor requer uma compreensão integral sobre a fisiologia humana em consonância com a história de vida, antecedentes familiares, processos de aprendizagem e ambiente sociocultural, dentre outros (Frutuoso \& Cruz, 2004).

O reconhecimento da dor a partir das escalas de avaliação, o conhecimento técnico-científico acerca da fisiopatologia da dor e do arsenal terapêutico disponível, a sensibilidade para o problema e a mudança de comportamento no sentido de uma abordagem multidisciplinar são de suma importância para minimizar o sofrimento enfrentado por esses pacientes (Araújo et al., 2012).

A técnica de acupuntura consiste na introdução de pequenas agulhas na pele, provocando respostas do organismo por meio dos estímulos nociceptivos. O controle da dor por meio da acupuntura ocorre mediante a estimulação cutânea tátil, que regula o influxo nociceptivos através de fibras finas e grossas. A fibra fina transmite o impulso da dor até as células T, já as fibras grossas funcionam como mecanismo de inibição da dor (Soáres, 2017).

Assim, a acupuntura promove uma hiperestimulação das fibras grossas, auxiliando o cérebro a liberar endorfina, um neurotransmissor natural que atua diminuindo a liberação da substância $\mathrm{P}$ (responsável pela resposta da dor) através da terminação da fibra fina (Machado et al., 2017). Nesse sentido, a acupuntura pode ser utilizada para tratar diferentes doenças, principalmente as relacionadas com alterações musculoesqueléticas (Perrupato \& Quirino, 2014), além disso, regula as funções orgânicas, endócrinas, autônomas e mentais, dentre outros benefícios. 
A dor é apresentada em 58\% a $80 \%$ dos casos em adultos internados para tratamento de câncer. Nas crianças, a prevalência ocorre em $78 \%$ dos casos durante diagnóstico, entre $25 \%$ e $58 \%$ no decorrer do tratamento e em até $90 \%$ na fase terminal da doença (Twycross et al_, 2015). Visto que a dor perpassa todas as fases do tratamento, ressalta-se a importância de associar, por meio da prática baseada em evidência, terapias complementares como a acupuntura para auxiliar o manejo da dor em pacientes oncológicos.

Considerando-se a problemática explicitada acima, objetiva-se com essa revisão identificar e analisar as evidências científicas do uso da acupuntura para alívio da dor em pacientes pediátricos oncológicos.

\section{Metodologia}

\section{Tipo de Estudo}

Trata-se de uma revisão integrativa que analisou a produção científica sobre a utilização da -acupuntura no alívio da dor em crianças e adolescentes com câncer. A presente revisão seguiu as etapas sugeridas na literatura (Botelho et al., 2011): 1) seleção da questão norteadora; 2) definição das características das pesquisas primárias da amostra; 3) seleção das pesquisas que compuseram a amostra da revisão; 4) análise dos achados dos artigos incluídos; 5) interpretação dos resultados; e 6) relato da revisão.

A pergunta de pesquisa foi formulada utilizando-se a estratégia PICO (Santos et al., 2007). A população (P) refere-se a crianças e adolescentes, a intervenção (I) é a acupuntura e o desfecho (O - outcome) é o alívio ou não da dor. Foi formulada a seguinte pergunta de pesquisa: Quais as evidências do uso da acupuntura para alívio da dor em crianças e adolescentes com câncer?

\section{Estratégia de Busca}

Para a busca e seleção dos artigos, foram consultadas as seguintes bases de dados: Medical Literature Analyses and Retrieval System Online (MEDLINE) via Pubmed, EMBASE, Literatura Latino Americana e do Caribe em Ciências da Saúde (LILACS) via Biblioteca Virtual em Saúde (BVS) e Cumulative Index to Nursing and Allied Health Literature (CINAHL).

A operacionalização desta pesquisa iniciou-se com consulta aos descritores em Ciências da Saúde (DECS) por meio da BVS, ao Medical Subject Headings (MESH) e ao título CINAHL também com descritores MESH. Utilizaram-se os seguintes descritores controlados, respeitando-se, contudo, as variações de cada base: "Acupuncture" OR "Acupuncture Therapy " OR "Acupuncture, Ear" OR "Acupuncture Points" OR "Acupuncture Analgesia" AND "Child" OR "Adolescent" OR "Infant" OR "Pediatrics" OR "Child" OR "Adolescent" OR "Youth" OR "Teen" OR "Infant" OR "Pediatric" OR "Paediatric" AND "Neoplasms" OR "Carcinoma" OR "Neoplas" OR "Cancer" OR "tumor" OR "Tumour" OR "Malignan" OR "Oncolog " AND "Palliative Care" OR "Palliative Medicine" OR "Terminal Care" OR "Palliative" OR "Supportive" OR "Terminal Care" OR "End of Life Care" OR "EOL Care" OR "Pain" OR "Acute Pain" OR "Pain Management" OR "Chronic Pain" OR "Ache" OR "Suffering".

A busca ocorreu nos meses de maio e junho de 2020 e a análise, entre julho e setembro de 2020. Foram utilizados como filtro: recorte temporal de 10 anos ( 2010 à 2020) e os idiomas inglês, português e espanhol. Justifica-se esse recorte temporal pela tentativa de alcançar mais estudos sobre a temática. Ressalta-se nessa etapa o valioso auxílio de um bibliotecário da área de ciências da saúde, o qual foi essencial para cruzar os descritores, combinando-os com os operadores booleanos $O R$ e AND. 


\section{Critérios de Seleção}

Foram adotados como critérios de inclusão: estudos originais (revisões sistemáticas, estudos clínicos randomizados e quase experimentais), estudos observacionais e relatos de casos. Foram excluídos: capítulos de livro, pesquisas ainda não concluídas e revisão de literatura.

\section{Análise e tratamento dos dados}

Dois revisores fizeram a revisão dos artigos de forma independente e os resultados foram semelhantes. Os estudos encontrados foram importados no software de gerenciamento de referências bibliográficas Endnote Web, disponibilizado na base Web of Science, com o intuito de ordenar os estudos e identificar os duplicados nas diferentes bases.

Para a extração e síntese das informações dos estudos selecionados, utilizou-se instrumento elaborado, proposto e validado por Ursi (Ursi \& Galvão, 2006), que apresenta os seguintes itens: identificação do artigo original, objetivos, características metodológicas do estudo, intervenções mensuradas, resultados encontrados e nível de evidência. Para a análise e posterior síntese dos artigos que atenderam aos critérios de inclusão, foi utilizado um quadro sinóptico elaborado também por Ursi e Galvão que contemplou os seguintes aspectos, considerados pertinentes: nome da pesquisa; nome dos autores; intervenção estudada; resultados; recomendações/conclusões (Ursi \& Galvão, 2006). Para a avaliação dos artigos quanto ao desenho da pesquisa, optou-se por seguir a classificação Grade (Ministério da Saúde, 2014) (Quadro 1).

Quadro 1. Níveis de evidências do delineamento da pesquisa de acordo com o sistema GRADE. Rio de Janeiro, RJ (2021).

\begin{tabular}{|l|l|l|l|}
\hline \multicolumn{1}{|c|}{ Nível } & \multicolumn{1}{|c|}{ Definição } & \multicolumn{1}{c|}{ Implicações } & \multicolumn{1}{c|}{ Fonte de informação } \\
\hline Alto & $\begin{array}{l}\text { Há forte confiança de } \\
\text { que o verdadeiro efeito } \\
\text { esteja próximo daquele } \\
\text { estimado. }\end{array}$ & $\begin{array}{l}\text { É improvável que trabalhos } \\
\text { adicionais irão modificar a } \\
\text { confiança na estimativa do efeito. }\end{array}$ & $\begin{array}{l}\text { - Ensaios clínicos bem delineados, com } \\
\text { amostra representativa. } \\
\text { - Em alguns casos, estudos } \\
\text { observacionais bem delineados, com } \\
\text { achados consistentes*. }\end{array}$ \\
\hline Moderado & $\begin{array}{l}\text { Há confiança } \\
\text { moderada no efeito } \\
\text { estimado. }\end{array}$ & $\begin{array}{l}\text { Trabalhos futuros poderão } \\
\text { modificar a confiança na estimativa } \\
\text { de efeito, podendo inclusive, } \\
\text { modificar a estimativa. }\end{array}$ & $\begin{array}{l}\text { - Ensaios clínicos limitados leves **. } \\
\text { com achados observacionais bem delineados, }\end{array}$ \\
\hline Baixo & $\begin{array}{l}\text { A confiança no efeito é } \\
\text { limitada. }\end{array}$ & $\begin{array}{l}\text { Trabalhos futuros provavelmente } \\
\text { terão um impacto importante em } \\
\text { nossa confiança na estimativa de } \\
\text { efeito. }\end{array}$ & $\begin{array}{l}\text { Ensaios clínicos com limitações } \\
\text { moderadas **. } \\
\text { - Estudos observacionais comparativos: } \\
\text { coorte e caso - controle. }\end{array}$ \\
\hline Muito Baixo & $\begin{array}{l}\text { A confiança na } \\
\text { estimativa de efeito é } \\
\text { muito limitada. Há um } \\
\text { incertante grau de }\end{array}$ & $\begin{array}{l}\text { Qualquer estimativa de efeito é } \\
\text { incerta }\end{array}$ & $\begin{array}{l}\text { - Ensaios clínicos com limitações } \\
\text { graves**. } \\
\text { - Estudos observacionais comparativos } \\
\text { presença de limitaçóes**. } \\
\text { - Estudos observacionais não comparados } \\
* * * . \\
\text { - Opiniões de especialistas. }\end{array}$ \\
\hline
\end{tabular}

*Estudos de coorte sem limitações metodológicas, com achados consistentes apresentando tamanho de efeito grande e/ou gradiente dose resposta. **Limitações: vieses no delineamento do estudo, inconsistência nos resultados, desfechos substitutos ou validade externa comprometida. ***Séries e relatos de casos.

Fonte: Elaboração GRADE Working Group.

\section{Aspectos éticos}

Por tratar-se de revisão integrativa e não envolver seres humanos, a pesquisa não foi submetida ao Comitê de Ética em Pesquisa. 


\section{Resultados e Discussão}

A partir dos critérios delimitados acima, a busca na base dados resultou em 82 artigos: sendo 24 na PUBMED, EMBASE - 38, LILACS/BVS - 1 e CINAHL - 19. Após os critérios de inclusão e exclusão, foram considerados como elegíveis para a amostra desta revisão 5 artigos. Para a seleção das publicações, seguiram-se as recomendações do Preferred Reporting Items for Systematic Reviews and Meta-Analyses (PRISMA) (Galvão et al., 2015), conforme apresentado na Figura 1.

Figura 1. Fluxograma de seleção dos artigos. Rio de Janeiro, RJ (2021).

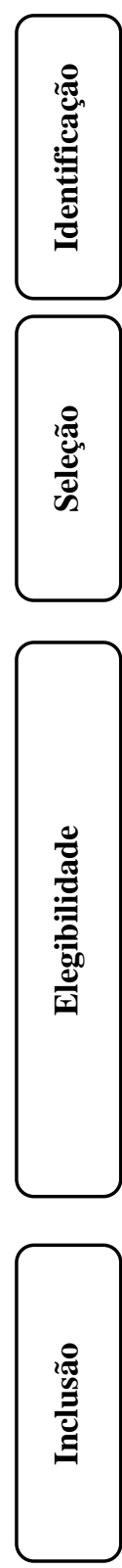

Número de estudos identificados nas bases de dados após filtros $\mathrm{N}=\mathbf{8 2}$

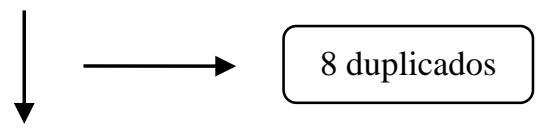

Número de estudos identificados:

$\mathbf{N}=\mathbf{7 4}$

Número de estudos selecionados: $\mathbf{N}=\mathbf{7 4}$

Estudos incluídos na revisão integrativa

$\mathrm{N}=10$

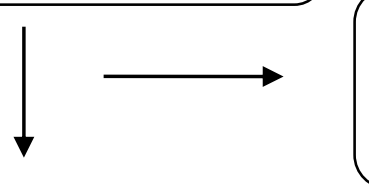

Estudos excluídos com base nos critérios adotados $\mathrm{N}=\mathbf{0 5}$

Número de estudos incluídos na revisão integrativa $\mathrm{N}=5$

Fonte: Adaptação da Recomendação PRISMA (Galvão et al., 2015).

Dos artigos selecionados, o mais recente data de 2018 e o mais antigo de 2013. Sendo três realizados nos Estados Unidos, um na Croácia e um da Áustria. Com relação à origem dos estudos, todos foram publicados no idioma inglês e em periódicos internacionais, sendo três em periódico específico de acupuntura, um em periódico médico e um em revista específica de oncologia. Quanto ao método, dois são relatos de caso, um estudo observacional e dois estudos quase experimentais (pré e pós-teste). No que se refere ao nível de evidência, um artigo mostrou nível de evidência baixa e quatro artigos nível muito baixo (Quadro 2). 
Research, Society and Development, v. 11, n. 1, e22911123311, 2022

(CC BY 4.0) | ISSN 2525-3409 | DOI: http://dx.doi.org/10.33448/rsd-v11i1.23311

Quadro 2. Nível de evidência de acordo com desenho de pesquisa do GRADE (Galvão et al., 2015). Rio de Janeiro, RJ (2021).

\begin{tabular}{|c|l|l|l|l|}
\hline Estudo & \multicolumn{1}{|c|}{ Autor /Ano } & \multicolumn{1}{|c|}{ País } & \multicolumn{1}{|c|}{ Desenho do estudo } & Nível de evidência \\
\hline E1 & Butkovic \& e Tot: (2018) & Croácia & Relato de caso & Muito baixo \\
\hline E2 & Chokshi et al.--(2017) & EUA & Estudo Observacional (comparativo) & Baixo \\
\hline E3 & Kurath-Koller et al.-, (2015) & Áustria & Relato de caso & Muito baixo \\
\hline E4 & Meyer et al.-; (2014) & EUA & Estudo quase experimental & Muito baixo \\
\hline E5 & Moisander-Joyce et al.-; (2013) & EUA & Estudo quase experimental & Muito Baixo \\
\hline
\end{tabular}

Fonte: Autores.

A caracterização dos estudos está descrita no Quadro 3 e organizada de acordo com o tipo de estudo, título, autor, país, objetivos e resultados. 
Quadro 3. Características dos estudos incluídos: ano, tipo de estudo, título, autor, país, objetivos e resultados. Rio de Janeiro, RJ (2021).

\begin{tabular}{|c|c|c|c|c|c|}
\hline Estudo & Tipo & Título/ Autor & País & Objetivos & Resultados \\
\hline E1 & Relato de caso & $\begin{array}{l}\text { Laser acupuncture } \\
\text { treatment of } \\
\text { neuropathic pain in a } \\
\text { boy with brain tumor } \\
\text { Butkovic \& e Tot. } \\
\text { (2018) } \\
\text { Butkovic \& Tot } \\
\text { (2018) }\end{array}$ & Croácia & $\begin{array}{l}\text { Uso de laser acupuntura para } \\
\text { um garoto de } 14 \text { anos com } \\
\text { tumor cerebral tratado com } \\
\text { cirurgia e recidivado. } \\
\text { Apresentou, após longa } \\
\text { internação em CTI, uma dor } \\
\text { neuropática nos MMII que o } \\
\text { impossibilitava a deambular. } \\
\text { Dor } 9,10 \text { pela escala visual } \\
\text { analógica. }\end{array}$ & $\begin{array}{l}\text { A laser acupuntura foi feita a cada } 3 \\
\text { dias totalizando } 10 \text { sessões. } \\
\text { Associada aos analgésicos já } \\
\text { utilizados. Após } 5 \text { sessões, } \\
\text { conseguiu usar meias. Após } 2 \text { meses, } \\
\text { conseguiu diminuir } \\
\text { progressivamente os analgésicos. } \\
\text { Após } 6 \text { meses, conseguia realizar } \\
\text { suas atividades normais e não mais } \\
\text { se queixava de dor neuropática. }\end{array}$ \\
\hline E2 & $\begin{array}{l}\text { Estudo } \\
\text { observacional } \\
\text { prospectivo }\end{array}$ & $\begin{array}{l}\text { Predictors of } \\
\text { acupuncture use } \\
\text { among children and } \\
\text { adolescents with } \\
\text { cancer } \\
\text { Chokshi et al. (2017) }\end{array}$ & EUA & $\begin{array}{l}\text { Noventa crianças } \\
\text { adolescentes que faziam } \\
\text { tratamento para câncer foram } \\
\text { acompanhados e receberam } \\
\text { acupuntura para alívio de } \\
\text { sintomas provenientes do } \\
\text { tratamento e da doença no } \\
\text { Centro Médico Universitário } \\
\text { da Columbia. Pacientes eram } \\
\text { acompanhados em casa e } \\
\text { quando internavam seguiam } \\
\text { com a acupuntura durante a } \\
\text { internação. }\end{array}$ & $\begin{array}{l}\text { A acupuntura teve seu uso com mais } \\
\text { frequência para dor, náusea, vômito } \\
\text { e xerostomia (boca seca), } \\
\text { dificuldade de concentração, cefaleia } \\
\text { e parestesia. Os resultados do estudo } \\
\text { abordaram a caracterização, } \\
\text { aceitação e satisfação dos } \\
\text { participantes. Concluíram que a } \\
\text { aceitação por parte das crianças e } \\
\text { adolescentes foi boa. Classificaram a } \\
\text { acupuntura como uma terapia segura } \\
\text { e recomendaram estudos clínicos } \\
\text { voltados para os variados sintomas } \\
\text { para validar seu uso na pediatria } \\
\text { oncológica }\end{array}$ \\
\hline E3 & $\begin{array}{l}\text { Relato de } \\
\text { caso }\end{array}$ & $\begin{array}{l}\text { Use of NADA ear } \\
\text { acupuncture in an } \\
\text { adolescent patient } \\
\text { with phantom limb } \\
\text { pain after surgery for } \\
\text { osteossarcoma } \\
\text { Kurath-Koller et al. } \\
\text { (2015) }\end{array}$ & Áustria & $\begin{array}{l}\text { Paciente de } 16 \text { anos com } \\
\text { osteossarcoma } \\
\text { após amputação, dor fantasma } \\
\text { de difícil manejo. } \\
\text { Foi } \\
\text { auriculoacupuntura para alívio } \\
\text { da dor e também ansiedade e } \\
\text { distúrbio do sono. }\end{array}$ & $\begin{array}{l}\text { Total de } 12 \text { sessões aplicadas em } 6 \\
\text { semanas. Antes da acupuntura foi } \\
\text { aplicado um questionário onde } \\
\text { avaliaram a dor e o bem estar geral - } \\
\text { em ambos foi obtido score de }(6 / 6) \text {. } \\
\text { Após o tratamento com a terapia } \\
\text { complementar, o score dor foi } 1 / 6 \text { e } \\
\text { do bem- estar geral } 2 / 6 \text {. }\end{array}$ \\
\hline $\mathrm{E} 4$ & $\begin{array}{l}\text { Estudo quase } \\
\text { experimental } \\
\text { (tipo pré e pós } \\
\text { teste) }\end{array}$ & $\begin{array}{l}\text { Examining the } \\
\text { association between } \\
\text { parental stress related } \\
\text { to child illness and } \\
\text { child pain across } \\
\text { acupuncture } \\
\text { treatments } \\
\text { Meyer et al. (2014) }\end{array}$ & EUA & $\begin{array}{l}\text { Este estudo examinou se a } \\
\text { acupuntura pode ajudar a } \\
\text { reduzir a dor infantil e } \\
\text { consequentemente o estresse } \\
\text { dos pais. } \\
29 \text { crianças com dores crônicas } \\
\text { participaram incluindo } \\
\text { paciente com leucemia. }\end{array}$ & $\begin{array}{l}\text { A dor infantil e o estresse dos pais } \\
\text { diminuíram significativamente após } \\
\text { dois tratamentos de acupuntura. }\end{array}$ \\
\hline $\begin{array}{c}\text { E5 } \\
2013\end{array}$ & $\begin{array}{l}\text { Estudo quase } \\
\text { experimental } \\
\text { (tipo pré e pós } \\
\text { teste) }\end{array}$ & $\begin{array}{l}\text { Children, adolescents } \\
\text { and young adults } \\
\text { (AYA) undergoing } \\
\text { treatment for cancer } \\
\text { perceive benefits to } \\
\text { acupuncture therapy } \\
\text { Moisander-Joyce et al. } \\
\text { (2013) }\end{array}$ & EUA & $\begin{array}{l}\text { Estudo avaliou a aceitação, } \\
\text { queixas principais de dor e } \\
\text { percepção da eficácia da } \\
\text { terapia em crianças, } \\
\text { adolescentes e adultos jovens } \\
\text { com câncer que aceitaram } \\
\text { receber a acupuntura. }\end{array}$ & $\begin{array}{l}\text { Crianças e pacientes em tratamento } \\
\text { contra o câncer acham a acupuntura } \\
\text { benéfica ao abordar os sintomas } \\
\text { relacionados ao câncer (por } \\
\text { exemplo, dor) e os efeitos colaterais } \\
\text { da quimioterapia (por exemplo, } \\
\text { náusea). }\end{array}$ \\
\hline
\end{tabular}


Quanto ao tipo de câncer, apenas os estudos E1 e E3 especificaram o tipo de tumor, sendo tumor cerebral e osteossarcoma, respectivamente.

Dos cinco estudos avaliados, três (E1, E3, E4) tiveram avaliação por meio de escalas, sendo a escala visual analógica (VAS) e o instrumento MyCaW (Measure Yourself Concerns and Wellbeing) para avaliação de preocupações e bem_-estar do paciente e a escala de dor com faces. Os outros 2 (E2, E5) não utilizaram escalas para avaliação da dor, mas questionários que abordavam mais subjetivamente a dor, a percepção das crianças em relação à acupuntura e aceitação em relação a essa terapia complementar (Quadro 3).

O estudo E3 realizou 12 sessões de acupuntura aplicadas em aproximadamente 6 semanas, utilizando a auriculoacupuntura com agulha. $\mathrm{O}$ estudo E1 utilizou a laser acupuntura a cada três dias durante 4 semanas, totalizando 10 sessões, e o estudo E4 utilizou agulhas e 8 sessões de acupuntura em um intervalo de aproximadamente 2 semanas.

O estudo E4 é o único que não diz respeito somente a pacientes com câncer, pois o estudo incluiu crianças e adolescentes com leucemia linfoide, espondilite anquilosante, neurofibromatose e malformação de Chiari. Os estudos E2 e E5 foram observacionais e também utilizaram agulha para realizar a acupuntura. Em se tratando do método, 2 são relatos de casos, 2 estudos quase experimentais e 1 estudo observacional.

Ressalta-se o fato de que nenhum estudo clínico foi encontrado nesta revisão, sendo este tipo de estudo que garantem melhor possibilidade de evidência científica.

A sensação da dor é uma experiência individual e muito pessoal do ser humano, manifestando-se mediante uma resposta fisiológica. É também um fenômeno emocional que conduz a um comportamento de fuga e proteção; deve ser entendida como um fenômeno muito complexo, afetado por variáveis biológicas, intelectuais, emocionais, espirituais e culturais (Siqueira et al., 2015).

Os aspectos psicológicos envolvidos na dor também têm papel de destaque na configuração geral do fenômeno. A característica multidimensional da dor requer uma compreensão integral sobre a fisiologia humana em consonância com a história de vida, antecedentes familiares, processos de aprendizagem, ambiente sociocultural, dentre outros (Frutuoso \& Cruz, 2004; Siqueira et al., 2015).

Estudos relatam que durante o tratamento do câncer as causas de dor variam e que esse sintoma está entre os mais relatados por crianças, adolescentes e por seus pais (Erickson et al., 2013; Kestler \& LoBiondo-Wood, 2012). Com relação à intensidade, varia de moderada a grave (Linder et al., 2018; Madi \& Clinton, 2018; Olagunju et al., 2016; Williams et al., 2015). Quanto à localização, a testa foi o local mais frequentemente citado seguido de dor no abdômen e dor na região inferior das costas (Madi \& Clinton, 2018).

Um dado importante publicado por Friedrichsdorf et al. (2007) é o relato de dor tipo breakthrough ou dor irruptiva oncológica feito por crianças e adolescentes. Esta se caracteriza por sua exacerbação em pacientes com dor basal estabilizada e que já recebem terapia com opioides (Saini et al., 2013). É uma dor grave ou incapacitante que atinge pico de intensidade minutos após o seu início e cessa geralmente entre 15 e 30 minutos. No referido estudo, metade das crianças relatam esse tipo de dor e a descrevem como uma dor em pontada ou como um tiro (Friedrichsdorf et al., 2007). Ressalta-se, assim, a importância da acupuntura como forma complementar para alívio da dor, visto ser essa uma queixa predominante em pacientes pediátricos com diagnóstico de câncer.

Um estudo realizado na Holanda examinou os sintomas físicos de crianças com câncer durante os cuidados paliativos. Foi realizado com 59 pais de crianças que faleceram neste período. Os sintomas físicos mais frequentes nas crianças (de acordo com o relato dos responsáveis) foram: dor, inapetência, fadiga, êmese e dificuldade na mobilidade (Theunissen et al., 2007). Percebe-se como a dor é uma das queixas mais constantes em crianças com câncer, o que vem reforçar e justificar os estudos voltados para esse sintoma. 
Jindal et al. (2008) relatam que tanto adultos quanto crianças com câncer podem ser afetados pela dor, porém, os principais estudos clínicos randomizados são referentes a adultos. Esta afirmação pode ser confirmada nos poucos artigos encontrados na presente revisão integrativa relacionando a acupuntura e a dor em crianças com câncer. A manifestação da dor em adultos e crianças ocorre de modo diferente, por isso, os resultados de estudos aplicados a adultos não podem ser extrapolados para as crianças. Os autores referem que mais estudos clínicos randomizados associando a acupuntura aos diversos problemas infantis, incluindo a dor, e também abordando a sua segurança devem ser realizados (Jindal et al., 2008).

Vários estudos relatam que a utilização de alguns quimioterápicos como platinas, taxanos e alcaloides da vinca podem promover a Dor Neuropática (DN), a qual pode persistir por meses ou anos após o uso (Bennett et al., 2012, Caponero et al., 2016; Schuler \& Heller, 2017). A DN é definida pelo Neuropathic Pain Special Interest Group da IASP como "sendo aquela decorrente de lesão ou doença que afeta diretamente o sistema somatossensitivo" (Miranda et al., 2016, p.2). Embora a semiologia da DN na infância possa ser comparável à do adulto, sua etiologia muitas vezes é diferente, com o agravante de que as opções terapêuticas são mais limitadas inclusive pela falta de estudos envolvendo a população pediátrica (Toste et al., 2015). O seu tratamento deve ser multimodal, incluindo o farmacológico e também as terapias complementares como a acupuntura, de forma a proporcionar um tratamento integral a crianças (Serrano et al., 2016).

Pode-se verificar o uso da acupuntura na dor neuropática no relato de caso do estudo E1. Um adolescente de 14 anos com tumor cerebral, após tratamento oncológico, inicia com uma dor neuropática em membros inferiores que o impossibilitava de andar, ficar de pé e -usar meias. -Paralelamente às medicações analgésicas, foi associada à auriculoacupuntura como terapia complementar. Após receber 5 sessões da auriculoacupuntura, o paciente conseguiu usar meias e após 2 meses de terapia, ele conseguiu ficar de pé e diminuir o uso de analgésicos. De acordo com este relato de caso, pode-se constatar como a acupuntura foi importante, conseguindo aliviar a dor do adolescente e consequentemente melhorar sua qualidade de vida. A auriculoacupuntura já foi testada em pacientes oncológicos adultos e mostrou resultados significativos na redução da dor e na diminuição do consumo de medicamentos (Ruela et al., 2018).

Um estudo de Tutelman et al. (2018) abordou a temática da dor e as estratégias farmacológicas, físicas e psicológicas utilizadas por pais e cuidadores para gerenciá-la nos seus filhos com diagnóstico de câncer. Após entrevistarem 230 responsáveis/cuidadores, concluíram que, apesar dos pais lançarem mão de estratégias para gerenciamento da dor, esta ainda continua sendo um problema para crianças que fazem tratamento oncológico. Isso demonstra o quão importante são as práticas integrativas e complementares e a necessidade de mais estudos relacionados à acupuntura no alívio da dor oncológica em pacientes pediátricos.

A presente revisão integrativa traz nos estudos E2 e E5 o uso da acupuntura para as principais queixas de crianças com câncer sendo a dor uma delas, o que corrobora com o estudo de Tutelman et al. (2018) de que este é um dos sintomas que mais afligem esses pacientes e seus familiares. Todavia, vale ressaltar que outros sintomas podem estar presentes como a náuseas, anorexia e fadiga (Moisander-Joyce et al., 2013).

Uma coorte realizada com crianças com anemia falciforme em uma instituição em Washington (EUA) acompanhou o tratamento com acupuntura voltado para a dor desses pacientes. Concluíram, a partir do relato em prontuário, que houve uma diminuição do score de dor, a qual foi bem tolerada, e que pacientes e responsáveis (em sua maioria) afirmaram querer usar a acupuntura em internações futuras (Mahmood, 2020). Mais um estudo que confirma o alívio da dor pela acupuntura, além da viabilidade e aceitabilidade tanto por pacientes quanto por seus responsáveis.

O estudo E3 relata sobre um paciente de 14 anos que, após uma amputação, sofre de uma dor fantasma de difícil controle. Após 12 aplicações de auriculoacupuntura, houve o alívio desta dor e a melhora do bem-estar do paciente. A dor fantasma que acomete muitos pacientes após desarticulação óssea foi avaliada em 25 pacientes que tiveram algum membro amputado no hospital St. Jude Children`s. No período de um ano, 76\% dos pacientes pediátricos apresentaram dor fantasma em 
algum momento (Burgoyne et al., 2012), demonstrando que esse tipo de dor tem papel relevante e merece atenção quanto à busca de meios para contê-la.

Dos cincos estudos avaliados, apenas o E1 relata o uso do laser para acupuntura. O laser tem sido mais uma opção para realizar a acupuntura e, em se tratando de crianças, é seguro, não causa a dor, não é invasivo e, se usado corretamente, não corre o risco de apresentar efeitos colaterais (Putri et al., 2020).

Entre os estudos selecionados, o E4, além de avaliar os pacientes, expandiu a acupuntura para os responsáveis das crianças e adolescentes. A dificuldade parental em lidar com os múltiplos aspectos da doença oncológica pode ter um impacto significativo na dor infantil e vice-versa. Mesmo que o tratamento da dor possa diminuir as dificuldades que os pais sentem, pode ser difícil eliminá-las completamente enquanto a criança estiver doente, influenciando na forma como o paciente pediátrico percebe sua dor. Por isso, achar uma maneira de amenizar o estresse que familiares sentem é importante para ajudar a aliviar a dor da criança e do adolescente e promover a sua saúde mental (Meyer et al., 2014).

Uma revisão sobre o uso da acupuntura na pediatria, conduzida por Lin \& Tung (2019), mostra que essa técnica é bem tolerada por crianças e adolescentes e que apresenta resultados positivos. Como frequência maior, apresenta-se o uso para cefaleias, dores abdominais, cólica de lactentes e para determinados procedimentos hospitalares que causam dor. Os autores concluem que o interesse pela acupuntura voltada para alívio da dor tem aumentado, porém, mais estudos clínicos randomizados devem ser realizados a fim de comprovar sua eficácia.

Frutuoso e Cruz (2004) e Siqueira et al. (2015) ressaltam que os aspectos psicológicos envolvidos na dor também têm papel de destaque na configuração geral do fenômeno. A característica multidimensional da dor requer uma compreensão integral sobre a fisiologia humana em consonância com a história de vida, antecedentes familiares, processos de aprendizagem e ambiente sociocultural, dentre outros.

Observa-se no estudo E4 que a acupuntura pôde auxiliar tanto os pacientes como também seus responsáveis, demonstrando que os benefícios desta terapia podem se estender aos familiares e o quanto isso pode impactar de forma positiva no bem-estar do paciente pediátrico.

Os resultados da presente revisão integrativa indicam estudos que utilizaram a acupuntura, seja com laser ou com agulha, para uma das principais queixas de crianças em tratamento oncológico: a dor. Nota-se um número baixíssimo de artigos encontrados e que nenhum apresenta alto nível de evidência. Esses achados revelam a necessidade da realização de mais estudos sobre a acupuntura voltados para essa temática. Todavia, diante dos resultados positivos dos 5 estudos, conclui-se o quanto essa prática integrativa e complementar pode contribuir para aliviar a dor e melhorar a qualidade de vida de crianças e adolescentes com câncer.

Como limitação desse estudo, está o critério de inclusão de artigos nos idiomas português, inglês e espanhol. Tal fato pode ter interferido nos achados de artigos importantes que respondessem à pergunta de pesquisa.

\section{Conclusão}

O número de estudos encontrados sobre o uso da acupuntura para a dor em crianças com câncer foi limitado. Apesar dos poucos artigos encontrados, todos indicaram resultados positivos para o alívio da dor com a utilização dessa técnica na oncologia pediátrica. Porém, ressalta-se o baixo nível de evidência dos métodos.

Essa revisão traz evidências de que a acupuntura, como terapia complementar pode trazer um benefício para a clientela pediátrica no que tange às queixas álgicas. Também nos evidencia que ela é uma prática viável no ambiente hospitalar e que tem boa aceitabilidade tanto por parte das crianças, adolescentes e de seus responsáveis.

Por isso, destaca-se a necessidade da realização de estudos com níveis de evidências mais contundentes, que comprovem e mostrem a eficácia desta prática. Sendo a dor considerada o quinto sinal vital e uma queixa tão frequente em 
pacientes oncológicos, é de suma importância a realização de estudos com delineamento de pesquisa mais sistemático para que a acupuntura possa ser inserida no planejamento do cuidado e utilizada como prática complementar para aliviar a dor de pacientes oncológicos pediátricos.

\section{Referências}

Araújo, M. C., Oliveira, M. B., \& Silva, Y. P. (2012). Pain assessment and treatment in children with oncological diseases. Revista Médica de Minas Gerais, 22, (Sup1 7), S22-S31.

Bennett, M. I., Rayment, C., Hjermstad, M., Aass, N., Caraceni, A., \& Kaasa, S. (2012). Prevalence and aetiology of neuropathic pain in cancer patients: a systematic review. PAIN, 153(2), 359-365.

Botelho, L. L. R., Cunha, C. C. A., \& Macedo, M. (2011). The integrative review method in organizational studies. Gestão e Sociedade, 5(11), 121-136.

Burgoyne, L. L., Billups, C. A., Jirón, J. L. Jr., Kaddoum, R. N., Wright, B. B., Bikhazi, G. B., et al. (2012). Phantom limb pain in young cancer-related amputees: recent experience at St Jude children's research hospital. Clinical Journal of Pain, 28(3), 222-225.

Butkovic, D., \& Tot, O. K. (2017). Laser acupuncture treatment of neuropathic pain in a boy with brain tumour. Complementary Therapies in Medicine, 35, 53-56.

Caponero, R., Montarroyos, E. S., \& Tahamtani, S. M. M. (2016). Post-chemotherapy neuropathy. Revista Dor, 17 (suppl 1), 56-58.

Chokshi, S. K., Ladas, E. J., Taromina, K., McDaniel, D., Rooney, D., Jin, Z., et al. (2017). Predictors of acupuncture use among children and adolescents with cancer. Pediatric Blood \& Cancer, 64(7), e26424.

Erickson, J. M., Macpherson, C. F., Ameringer, S., Baggott, C., Linder, L. A., \& Stegenga, K. (2013). Symptoms and symptom clusters in adolescents receiving cancer treatment: A review of the literature. International Journal of Nursing Studies, 50, 847-869.

Friedrichsdorf, S. J., Finney, D., Bergin, M., Stevens, M., \& Collins, J. J. (2007). Breakthrough pain in children with cancer. Journal of Pain and Symptom Management, 34(2), 209-16.

Frutuoso, J. T., \& Cruz, R. M. (2004). Relato verbal na avaliação psicológica da dor. Avaliação Psicológica, 3(2), $107-114$.

Galvão, T. F., Pansani, T. A. S., \& Harrad, D. (2015). Principais itens para relatar revisões sistemáticas e meta-análises: a recomendação PRISMA. Epidemiologia e Serviços de Saúde, 24(2), 335-342.

Gottschling, S., Meyer, S., Langler, A., Scharifi, G., Ebinger, F., \& Gronwald, B. (2014). Differences in use of complementary and alternative medicine between children and adolescents with cancer in Germany: a population based survey. Pediatric Blood \& Cancer, 61(3), 488-492.

Jindal, V., Ge, A., \& Mansky, P. J. (2008). Safety and efficacy of acupuncture in children: a review of the evidence. Journal of Pediatric Hematology/Oncology, 30(6), 431-442.

Kestler, S. A., \& LoBiondo-Wood, G. (2012). Review of symptom experiences in children and adolescents with cancer. Cancer Nursing, 35 , e31-e49.

Kurath-Koller, S., Bauchinger, S., Sperl, D., Leithner, A., Urlesberger, B., Raith, W., et al. (2014). Use of NADA ear acupuncture in an adolescent patient with phantom limb pain after surgery for osteosarcoma. Acupuncture in Medicine, 33(1), 822-824.

Lin, Y. C., Perez, S., \& Tung, C. (2019). Acupuncture for pediatric pain: The trend of evidence-based research. Journal of Traditional and Complementary Medicine, 10(4), 315-319.

Linder, L. A., Al-Qaaydeh, S., \& Donaldson, G. (2018). Symptom characteristics among hospitalized children and adolescents with cancer. Cancer Nursing, $41,32-32$.

Madi, D., \& Clinton, M. (2018). Pain and its impact on the functional ability in children treated at the Children's Cancer Center of Lebanon. Journal of Pediatric Nursing, 39, e11- e20.

Machado, C. L., Brito, R. M., \& Belmonte, M. L. (2017). Efeitos da aplicação da eletroestimulação nervosa transcutânea (TENS) sobre a dor e volumes pulmonares em indivíduos submetidos à cirurgia cardíaca. Revista Fisioterapia \& Reabilitação, 1(1), 34-41.

Mahmood, L. A., Reece-Stremtan, S., Idiokitas, R., Martin, B., Margulies, S., Hardy, S. J., Bost, J. E., \& Darbari, D. S. (2020). Acupuncture for pain management in children with sickle cell disease. Complementary Therapies in Medicine, 49, 102287.

Meyer, R. M. L., Barber, B. A., Kobylecka, M., \& Gold, J. I. (2014). Examining the association between parental stress related to child illness and child pain across acupuncture treatments. Medical Acupuncture, 26(1), 23-30.

Ministério da Saúde (2017). Secretaria de Atenção à Saúde. Protocolo de diagnóstico precoce do câncer pediátrico. Brasília: Ministério da Saúde. 
Ministério da Saúde (2014). Diretrizes metodológicas: sistema GRADE - manual de graduação da qualidade da evidência e força de recomendação para tomada de decisão em saúde. https://bvsms.saude.gov.br/bvs/publicacoes/diretrizes_metodologicas_sistema_grade.pdf

Miranda, C. C. V., Seda Junior, L. F., \& Pelloso, L. R. C. A. (2016). Nova classificação fisiológica das dores: o atual conceito de dor neuropática. Revista Dor, 17(supl. 1), 2-4.

Moisander-Joyce, H., Bombacie, M., Ladas, E., Taromina, K., Rooney, D., Chokshi, S., et al. (2013). Children, adolescents and young adults (AYA) undergoing treatment for cancer perceive benefits to acupuncture therapy. Journal of Alternative and Complementary Medicine, 19(7), 1-53.

Olagunju, A. T., Sarimiye, F. O., Olagunju, T. O., Habeebu, M. Y., \& Aina, O. F. (2016). Child's symptom burden and depressive symptoms among caregivers of children with cancers: An argument for early integration of pediatric palliative care. Annals of Palliative Medicine, 5, $157-165$.

Perrupato, T. F., \& Quirino, A. C. T. (2014). Acupuntura como terapia complementar no tratamento de displasia coxofemoral em cães - relato de caso. Revista de Ciência Veterinária e Saúde Pública, 1(2), 141-145.

Pontes, C. M., \& Kurashima, A. Y. (2009). Criança com câncer: revisão de literatura sobre sinais e sintomas presentes na fase de cuidados paliativos. Revista da Sociedade Brasileira de Enfermeiros Pediatras, 9(1), 27-31.

Putri, D. E., Srilestari, A., Abdurrohim, K., Mangunatmadja, I., \& Wahyuni, L. K. (2020). The effect of laser acupuncture on spasticity in children with spastic cerebral palsy. Journal of Acupuncture and Meridian Studies, 3(5), 152-156.

Raja, S. N., Carr, D. B., Cohen, M., Finnerup, N. B., Flor, H., Gibson, S., et al. (2020). The revised International Association for the Study of Pain definition of pain: concepts, challenges, and compromises. Pain. PAIN, 161 (9), 1976-1982.

Ruela, L. O., Iunes, D. H., Nogueira, D. A., Stefanello, J., \& Gradim, C. V. C. (2018). Effectiveness of auricular acupuncture in the treatment of cancer pain: randomized clinical trial. Revista da Escola de Enfermagem da USP, 52, e03402.

Saini, A., Tucci, M., Tampellini, M., Maina, D., Bouraouia, K., Giuliano, PL., et al (2013). Circadian variation of breakthrough pain in cancer patients. European Journal of Pain, 17(2), 264-70.

Sakiroglu, C. O., Wood, C., \& Cunin-Roy, C. Pain in adolescents with cancer. (2009). Bulletin du Cancer, 96(2), 37-45.

Santos, C. M. C., Pimenta, C. A. M., \& Nobre, M. R. C. (2007). The PICO strategy for the research question construction and evidence search. Revista LatinoAmericana de Enfermagem, 15(3), 508-511.

Schuler, U., \& Heller, S. (2017). Chemotherapy-induced peripheral neuropathy and neuropathic pain. Schmerz, 31(4), 413-425.

Serrano, S. C., Barbosa, S. M. M., Queiroz, E. J., Campos, F. G., \& Santos, A. P. S. V. (2016). Peculiaridades da dor neuropática na criança. Revista Dor, 17(supl. 1), 110-112.

Siqueira, H. B. O. M., Santos, M. A., Gomez, R. R. F., Saltareli, S., \& Sousa, F. A. E. F. (2015). Pain expression in children with cancer: a phenomenological comprehension. Estudos de Psicologia, 32(4), 663-674.

Soáres, L. M. C. (2017). Intervenção na diminuição da dor na punção intramuscular. Mestrado em educação para saúde [dissertação]. Coimbra: Instituto Politécnico de Coimbra, Escola Superior de Tecnologia da Saúde https://comum.rcaap.pt/bitstream/10400.26/22678/3/Le\%C3\%B4ncio\%20de\%20Matos\%20Coe\%20Soares.pdf

Theunissen, J. M. J., Hoogerbrugge, P. M., Van Achterberg, T., Prins, J. B., Vernooij-Dassen, M. J. F. J., \& Van Den Ende, C. H. M. (2007). Symptoms in the palliative phase of children with cancer. Pediatric Blood \& Cancer, 49, 160-165.

Toste, S., Palhau, L. I., \& Amorim, R. (2015). Neuropathic pain in children. Revista da Sociedade Portuguesa de Medicina Física e de Reabilitação, 27(1), $22-29$.

Tutelman, P. R., Chambers, C. T., Stinson, J. N., Parker, J. A., Fernandez, C. V., Witteman, H. O., et al. (2018). Pain in children with cancer: prevalence, characteristics, and parent management. Clinical Journal of Pain, 34(3), 198-206.

Twycross, A., Parker, R., Williams, A., \& Gibson, F. (2015). Cancer-related pain and pain management: sources, prevalence, and the experiences of children and parents. Journal of Pediatric Oncology Nursing, 32(6), 369-384.

Ursi, E. S., \& Gavão, C. M. (2006). Perioperative prevention of skin injury: an integrative literature review. Revista Latino-Americana de Enfermagem, 14(1), 124-131. 